\title{
Performance of a Permanent-Magnet Cylindrical Hall-Effect Thruster
}

\author{
K.A. Polzin, E.S. Sooby ${ }^{\dagger}$ and A.C. Kimberlin ${ }^{\ddagger}$ \\ NASA-Marshall Space Flight Center, Huntsville, AL 35812 \\ Y. Raitses $\$$ E. Merino and N.J. Fisch ${ }^{\uparrow}$ \\ Princeton Plasma Physics Laboratory, Princeton, NJ 08543
}

\begin{abstract}
The performance of a low-power cylindrical Hall thruster, which more readily lends itself to miniaturization and low-power operation than a conventional (annular) Hall thruster, was measured using a planar plasma probe and a thrust stand. The field in the cylindrical thruster was produced using permanent magnets, promising a power reduction over previous cylindrical thruster iterations that employed electromagnets to generate the required magnetic field topology. Two sets of ring-shaped permanent magnets are used, and two different field configurations can be produced by reorienting the poles of one magnet relative to the other. A plasma probe measuring ion flux in the plume is used to estimate the current utilization for the two magnetic topologies. The measurements indicate that electron transport is impeded much more effectively in one configuration, implying higher thrust efficiency. Thruster performance measurements on this configuration were obtained over a power range of $70-350 \mathrm{~W}$ and with the cathode orifice located at three different axial positions relative to the thruster exit plane. The thrust levels over this power range were 1.25-6.5 mN, with anode efficiencies and specific impulses spanning $4-21 \%$ and $400-1950 \mathrm{~s}$, respectively. The anode efficiency of the permanent-magnet thruster compares favorably with the efficiency of the electromagnet thruster when the power consumed by the electromagnets is taken into account.
\end{abstract}

\section{Introduction}

W ${ }^{\mathrm{HILE}}$ annular Hall thrusters can operate at high efficiency at $\mathrm{kW}$ power levels, it is difficult to construct one that operates over a broad envelope from $\sim 1 \mathrm{~kW}$ down to $\sim 100 \mathrm{~W}$ while maintaining an efficiency of $45-55 \%$. Scaling to low power while holding the main dimensionless parameters constant requires a decrease in the thruster channel size and an increase in the magnetic field strength. ${ }^{1,2}$ Increasing the magnetic field becomes technically challenging since the field can saturate the miniaturized inner components of the magnetic circuit and scaling down the magnetic circuit leaves very little room for magnetic pole pieces and heat shields. In addition, the central magnetic pole piece defining the interior wall of the annular channel can experience excessive heat loads in a miniaturized Hall thruster, with the temperature eventually exceeding the Curie temperature of the material ${ }^{2}$ and in extreme circumstances leading to accelerated erosion of the channel wall.

An alternative approach is to employ a cylindrical Hall thruster (CHT) geometry. ${ }^{3}$ Laboratory model CHTs have operated at power levels ranging from $\sim 50 \mathrm{~W}$ up to $\sim 1 \mathrm{~kW}$. These thrusters exhibit performance characteristics that are comparable to conventional, annular Hall thrusters of similar size. Compared to the annular Hall thruster, the CHT's insulator surface area to discharge chamber volume ratio is lower. Consequently, there is the potential for reduced wall losses in the channel of a CHT, and any reduction in wall losses should translate into lower channel heating rates and reduced erosion, making the CHT geometry promising for low-power applications. This potential for high performance in the low-power regime has served as the impetus for research and development efforts aimed at understanding and improving CHT performance. ${ }^{3-7}$

\footnotetext{
*Propulsion Research Engineer, Propulsion Research and Technology Applications Branch, Propulsion Systems Department. Senior Member AIAA.

†Undergraduate Intern; currently IGERT Applied Science Research Fellow, Texas A\&M University, College Station, TX. Member AIAA.

$\ddagger$ Co-op Student; currently Undergraduate Student, Tennessee Technological University, Cookeville, TN. Member AIAA.

$\S$ Research Physicist. Member AIAA.

TProfessor, Astrophysical Sciences Dept. Member AIAA.

This material is declared a work of the U.S. Government and is not subject to copyright protection in the United States.
} 
In this paper, we present results from tests performed with a CHT that uses permanent magnets to produce the magnetic field topology. This thruster has the promise of reduced overall power consumption over previous CHT iterations that employed electromagnets. The use of permanent magnets simplifies the design by removing the multiturn electromagnet coils from the thruster. Beam current data are presented to show the effect of the magnetic field topology on the plume profile and current utilization. Thruster performance measurements (thrust, $I_{\mathrm{sp}}$, and anode efficiency) obtained using a thrust stand are presented to evaluate against previous electromagnet CHT performance levels and also to provide a baseline against which future iterations of the permanent magnet CHT may be compared.

\section{Experimental Apparatus}

Tests were conducted on a cylindrical Hall thruster at both the Princeton Plasma Physics Laboratory (PPPL) and NASA's Marshall Space Flight Center (MSFC). We proceed first with a description of the thruster and then discuss the facilities at both PPPL and MSFC that were used to test the CHT.

\section{A. Cylindrical Hall Thruster with Permanent Magnets}

Measurements were obtained using the $2.6 \mathrm{~cm}$ channel diameter permanent magnet PPPL CHT shown in Fig. 1. The thruster is roughly $5.5 \mathrm{~cm}$ in overall diameter and $3.5 \mathrm{~cm}$ long, massing roughly $350 \mathrm{~g}$. The thruster channel is comprised of a ceramic boron-nitride insulator with propellant fed from an annular anode. Two sets of samarium-cobalt ( $\mathrm{Sm}-\mathrm{Co}$ ) rare-Earth magnets are used to produce the magnetic field. The magnets can be oriented in the same direction to produce the 'direct' magnetic field topology shown in Fig. 2a, or they can be oriented to oppose each other producing the 'cusp' field configuration in Fig. $2 b$. The magnetic field in the plume region is roughly the same for either permanent magnet configuration, but the field strength is greater in the direct-field configuration. There are also differences in the topology inside the thruster channel, with a greater axial component in the direct-field configuration. The maximum field strength inside the thruster channel in either configuration is roughly $1 \mathrm{kG}$.

The working propellant for all experiments is research-grade xenon gas, and the cathode and anode flow rates are independently controlled. A commercial HeatWave Labs HWPES-250 hollow cathode is used in these experiments, serving as both the thruster cathode and the beam neutralizer.

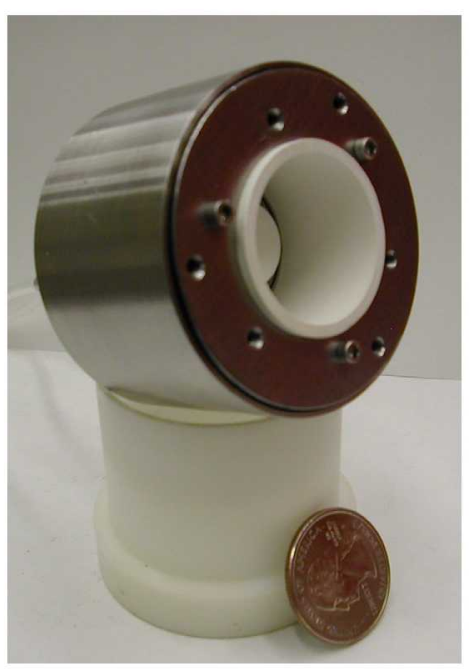

Figure 1. Laboratory model $2.6 \mathrm{~cm}$ CHT with Sm-Co permanent magnets (with US quarter for scale).

a)

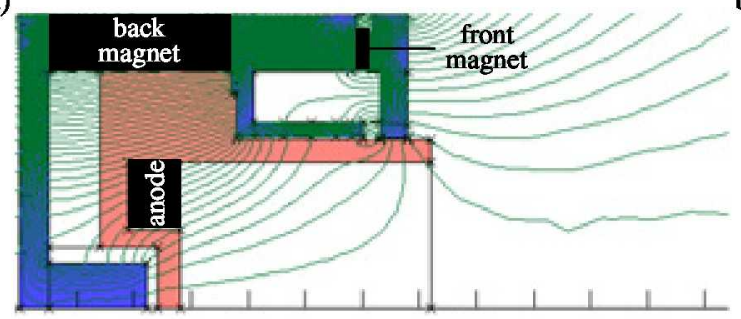

b)

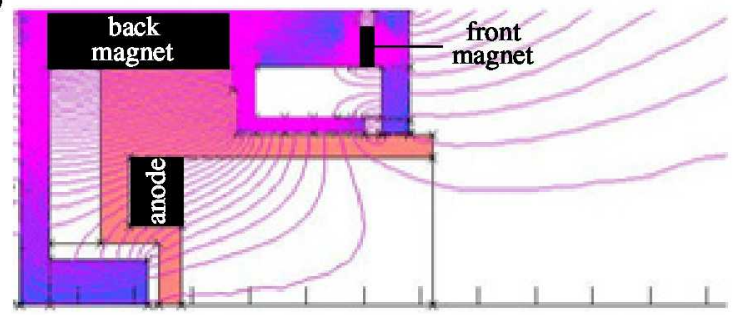

Figure 2. Magnetic field topology in a laboratory model $2.6 \mathrm{~cm}$ CHT with Sm-Co permanent magnets where the magnets are oriented in a) a direct-field alignment and b) a cusp-field alignment. The maximum magnetic field is roughly $1 \mathrm{kG}$ at the axis near the back wall.

\section{B. Princeton Test Facility}

Testing at Princeton was conducted in the PPPL large Hall thruster facility. ${ }^{8}$ The vacuum vessel has a volume of 28 $\mathrm{m}^{3}$ and is equipped with cryopumps that maintain the background pressure at a level that does not exceed $3 \times 10^{-6}$ torr. The angular ion flux distribution in the plume was measured using a $2.54 \mathrm{~cm}$ planar plume probe with guarding 

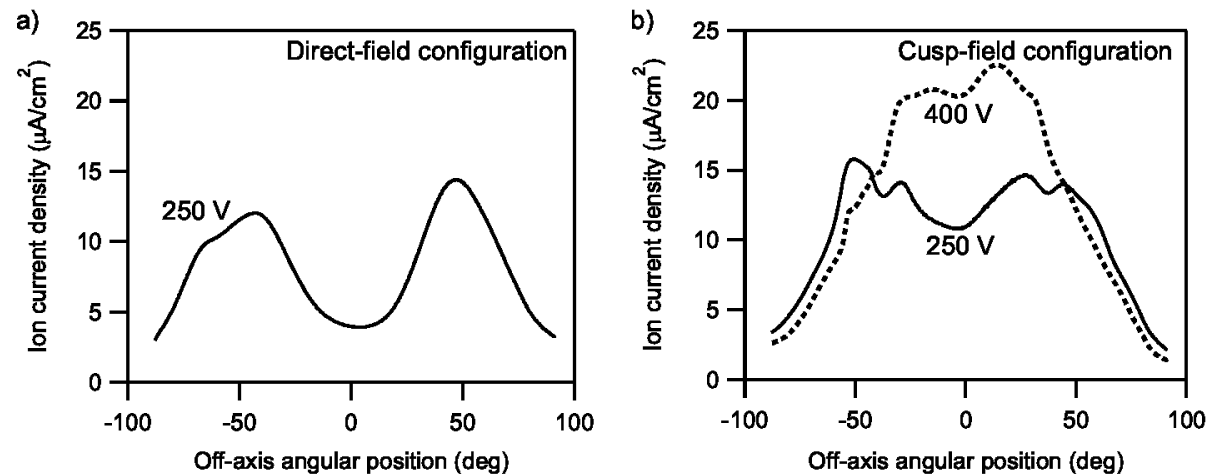

Figure 3. Planar plume probe measurements showing the ion flux distribution in the CHT beam as a function of off-axis angular position for a) the direct-field configuration and b) the cusp-field configuration. The voltages given for each data set represent the thruster discharge voltages. The anode flow rate for all plume measurements was $3.4 \mathrm{sccm}$.

Table 1. Summary of discharge and beam current data and current utilization for the direct and cusp-field configurations. The anode flow rate was $3.4 \mathrm{sccm}$.

\begin{tabular}{cccc}
\hline \hline Condition & Discharge Current $I_{d}(\mathrm{~A})$ & Ion Beam Current $I_{i}(\mathrm{~A})$ & Current Utilization $I_{i} / I_{d}$ \\
\hline Direct, 250 V & 0.35 & 0.24 & 0.69 \\
Cusp, 250 V & 0.59 & 0.28 & 0.47 \\
Cusp, 400 V & 0.56 & 0.30 & 0.54 \\
\hline \hline
\end{tabular}

rings like the type described in Refs. $[9,10]$. The probe is located $70 \mathrm{~cm}$ from the thruster and can be rotated through an angle of \pm 90 degrees relative to the thruster axis.

\section{NASA-MSFC Test Facility}

Testing at NASA-MSFC was conducted in a $2.75-\mathrm{m}$ diameter, $7.6-\mathrm{m}$ long stainless steel vacuum chamber. Previous testing of a PPPL CHT was also performed in this facility. ${ }^{6}$ The vacuum level inside the chamber is maintained by two $9500 \mathrm{l} / \mathrm{s}$ gaseous helium cryopumps. The base pressure of the facility was $2 \times 10^{-7}$ torr, and the pressure level during testing was roughly $1 \times 10^{-5}$ torr.

The propellant flow rate to both the cathode and anode were controlled using two variable 10-sccm MKS 1479 precision flow controllers (calibrated on Xe and controllable to $\pm 0.1 \mathrm{sccm}$ ). All testing was performed with a cathode flow rate of $2 \mathrm{sccm}$.

Thrust was measured using the variable-amplitude hanging pendulum with extended range (VAHPER) thrust stand. ${ }^{11}$ The stand employs a unique linkage mechanism to convert horizontal deflection of the pendulum arm into amplified vertical deflection of a secondary beam. Displacement (thrust) calibration of the VAHPER thrust stand is accomplished using an in situ calibration rig that applies a series of known loads normal to the pendulum arm. Calibration can be performed before, during, and after thruster operation. The measured displacement of the vertically deflecting linkage is recorded as the calibration loads are applied to the arm. Assuming that the relationship between the applied force and the measured displacement is linear allows for a linear curve fit of the calibration data.

The cathode is mounted on a stage that allows for axial translation relative to the thruster exit. In the reference position, the cathode exit is $1.9 \mathrm{~cm}(0.75$ ") downstream of the thruster exit and $5.1 \mathrm{~cm}$ (2") from the thruster centerline. The cathode is aligned such that its centerline forms a $40^{\circ}$ angle with the thruster centerline. The translation stage moves the cathode exit from the reference position to locations that are further downstream of the thruster.

\section{Experimental Data}

Data from testing conducted with the permanent-magnet CHT are presented in this section. Beam ion current density measurements obtained at PPPL are presented first and followed by performance measurements performed at NASA-MSFC. 


\section{A. Ion Current Density}

The plasma beam current measurements for both the direct and cusp magnetic field configurations with an anode flow rate of $3.4 \mathrm{sccm}$ are presented in Fig. 3. The beam current density in the direct-field configuration (Fig. 3A) is smaller than the current density in the cusp-field configuration (Fig. 3B). In addition, the ion flux near the centerline in the cusp-field configuration is increased when the thruster discharge voltage is increased from $250 \mathrm{~V}$ to $400 \mathrm{~V}$. The thruster discharge current $I_{d}$ and measured ion beam current $I_{i}$ are summarized in Table 1 for each of the magnetic field configuration/discharge voltage combinations tested. The current utilization $I_{i} / I_{d}$ for each case is also calculated in the table.

The cusp-field configuration ion beam current plume measurements shown in Fig. 3B exhibit a greater ion flux along the centerline as the discharge voltage is increased from 250 to $400 \mathrm{~V}$. A comparison with the direct-field configuration data in Fig. 3A indicates a somewhat increased ion flux in the plume for the cusp-field case.

The current utilization ratio in Table 1 is useful in determining how effectively the applied magnetic field suppresses electron transport to the anode. Holding all other parameters constant, thruster efficiency will decrease with increasing electron current. ${ }^{12}$ The high current utilization in the direct-field configuration almost certainly implies that electron transport is lowest in that case, leading to the conclusion that the thrust efficiency will be higher for this configuration than in the cusp-field case.

\section{B. Thruster Performance}

Anode efficiency, specific impulse $\left(I_{\mathrm{sp}}\right)$ and thrust measurements obtained for the direct-field configuration CHT are presented in Figs. 4 and 5. Anode efficiency and $I_{\mathrm{sp}}$ (specifically, anode $I_{\mathrm{sp}}$ ) are computed according to their standard definitions ${ }^{13}$ using the measured thrust level, the anode mass flow rate, and the power supply current and voltage readings.

The data in each set of graphs correspond to thrust measurements at anode mass flow rates of 3.4, 4.0, 4.4, and $5.0 \mathrm{sccm}$. The data in Fig. $4 \mathrm{a}-\mathrm{c}$ were obtained with the cathode exit orifice located at the base position cited previously in the text, while the data in Figs. 5a-c and $5 \mathrm{~d}-\mathrm{f}$ were obtained with the cathode translated axially downstream $1.25-\mathrm{cm}(0.5-\mathrm{in})$ and $2.5-\mathrm{cm}(1.0-\mathrm{in})$, respectively. The data span a range from 70 to $350 \mathrm{~W}$ in discharge power and show anode efficiencies between 4 and $21 \%, I_{\mathrm{sp}}$ levels between 400 and $1955 \mathrm{~s}$, and thrust levels from 1.25 to $6.5 \mathrm{mN}$. Higher power levels were attempted with the cathode translated axially downstream, but discharge instability precluded the acquisition of data at those positions.

The anode efficiency, anode $I_{\mathrm{sp}}$ and thrust all generally increase with increasing discharge power up to about $250 \mathrm{~W}$. Beyond that power level the $I_{\mathrm{sp}}$ and thrust continue to increase at roughly the same rate, but the efficiency begins to decrease. Axially translating the cathode does not appear to have a dramatic effect on performance, however for similar operating conditions (voltage and flow rate) the maximum discharge power that is accessible appears to be more limited. Anode flow rate, on the other hand, appears to have very little effect on the measured performance at higher power levels $(>140$ W). At power levels below $140 \mathrm{~W}$, the data exhibit considerable scatter from point to point. The data presented in this paper were obtained over the course of thruster operation over several days of testing.
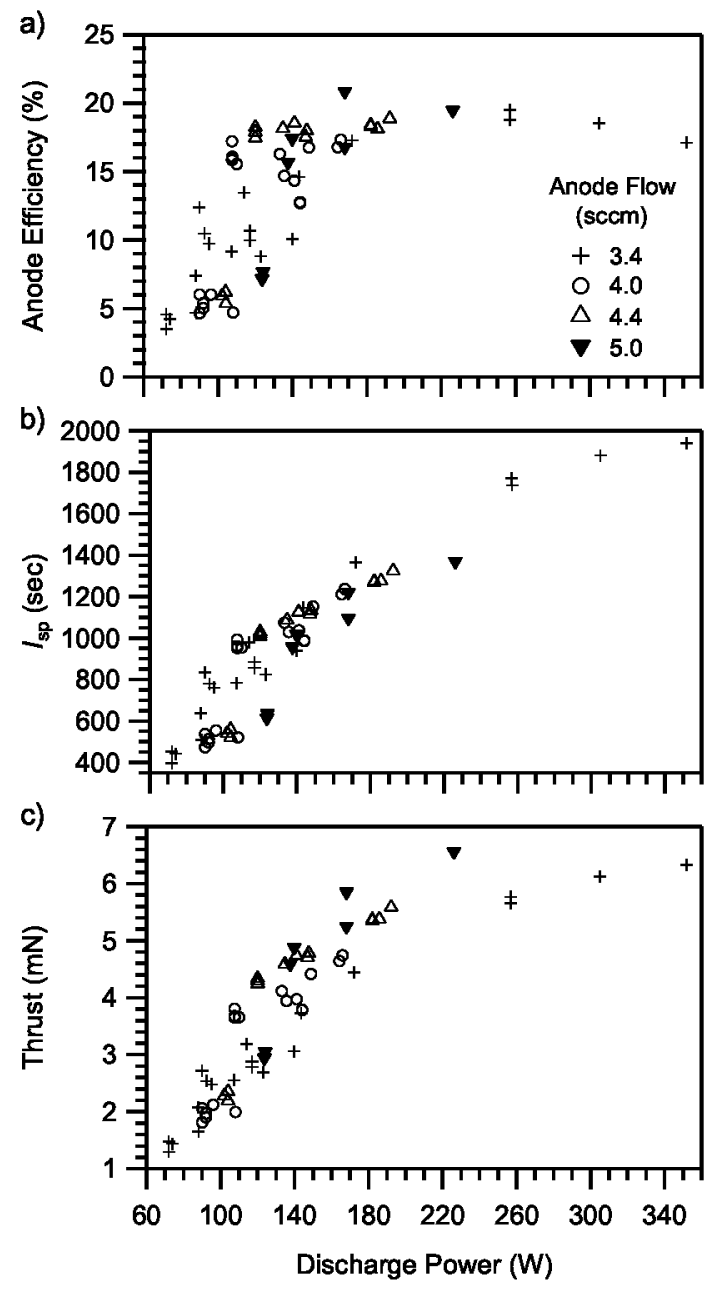

Figure 4. (a) Anode efficiency, (b) $I_{\mathrm{sp}}$, and (c) thrust as a function of discharge power obtained with the cathode at its base position. The average error bars (with a 95\% confidence interval) on these data are $1.1 \%, 44 \mathrm{sec}$, and $0.16 \mathrm{mN}$. 

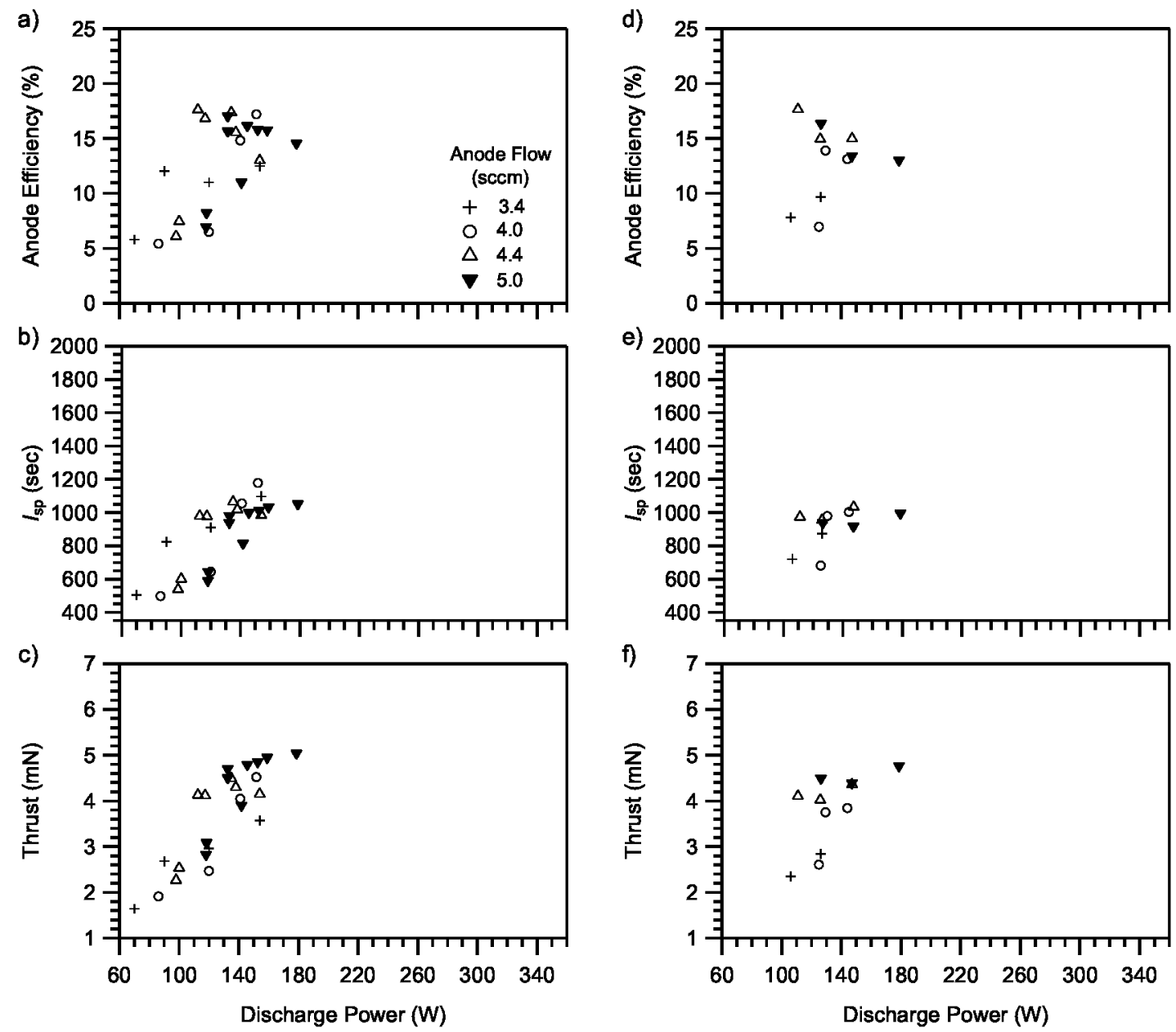

Figure 5. Anode efficiency, $I_{\mathrm{sp}}$, and thrust as a function of discharge power obtained with the cathode with the cathode linearly translated 1.25-cm (0.5-in) downstream from its base position; (a), (b), and (c), respectively; and linearly translated 2.5-cm (1.0-in) downstream from its base position; (d), (e), and (f), respectively. The average error bars (with a 95\% confidence interval) on these data are $1.1 \%, 44$ sec, and $0.16 \mathrm{mN}$.

The thruster would increase in temperature rapidly, quickly reaching a value above which operation could no longer be performed without endangering the magnets. Once the thruster was shut down and cooled sufficiently, operation could proceed again until the temperature limit was again reached. At low thruster temperatures (specifically, at the start of an operating cycle) the thruster sometimes drew an anomalously high level of current, consistent with a cathode or channel insulator surface in need of surface cleaning and conditioning. As the temperature rose, these current levels returned to their expected values. Data from these periods where the current was higher than normal are not included in this paper, but the existence does point to the importance of surface conditioning. The complete dataset showing the discharge current as a function of discharge voltage and anode flow rate are presented in Fig. 6 . We observe that discharge current generally increases with increasing mass flow rate.

Comparisons to performance values previously obtained for a $3-\mathrm{cm}$ PPPL CHT ${ }^{6}$ that employed electromagnet coils show that at $150 \mathrm{~W}$ the $I_{\mathrm{sp}}$ and efficiency in the permanent magnet thruster are both lower than those measured in the electromagnet thruster ( $I_{\mathrm{sp}}$ and anode efficiency reduced from $\sim 1400 \mathrm{~s}$ to $\sim 1100 \mathrm{~s}$ and $26 \%$ to $16-18 \%$, respectively). However, the anode efficiency of the 3-cm CHT does not account for the electrical power invested in the production of the magnetic field, which was on average roughly $100 \mathrm{~W}$. When this power is considered, the anode efficiency of the 3-cm CHT becomes $15.5 \%$, which is slightly less than the efficiency obtained on the permanent magnet thruster.

Data showing the anode efficiency and $I_{\mathrm{sp}}$ as a function of discharge voltage are presented in Fig. 7. In (a)-(d), we observe that the efficiency and specific impulse are both quite low at discharge voltages below $250 \mathrm{~V}$ (no data was acquired at lower discharge voltages when the cathode was linearly translated $2.5-\mathrm{cm}$ (1-in) downstream). From 250-350 V, performance increases significantly as the anode flow rate is adjusted from 3.4 to $4.0 \mathrm{sccm}$. Performance 
increases at greater flow rates are not readily discernable in the data set. Finally, we note that as the discharge voltage is taken from 400 to $550 \mathrm{~V}$, the efficiency peaks and actually begins to decrease. This is most likely associated with the increase in discharge current as a function of voltage (see Fig. 6a), which is due to a combination of an increase in electron current across the magnetic field and an increase in ion beam current as the voltage is raised.

\section{Conclusions}

Conventional (annular) Hall thrusters are efficient in the kilowatt power regime, but become inefficient at small sizes as the insulator surface area to discharge chamber volume ratio increases, leading to a commensurate increase in plasma wall losses. The CHT geometry eliminates the inner discharge channel surface and has a non-traditional magnetic field topology, giving it a lower insulator surface area to discharge chamber volume ratio and the promise of improved performance in the low power regime. This promise has in the past been demonstrated in a series of CHT designs that employed electromagnets to generate the magnetic field topology in the thruster.

In the present work, preliminary measurements were obtained on a 2.6-cm PPPL CHT that used permanent magnets to generate the applied magnetic field in the thruster. The use of permanent magnets promises even lower thruster power consumption by eliminating the electromagnet power sink. In addition, permanent magnets simplify the design, removing the need for additional power supplies and multi-tum electromagnet coils. While ion flux in the plume was somewhat higher for the

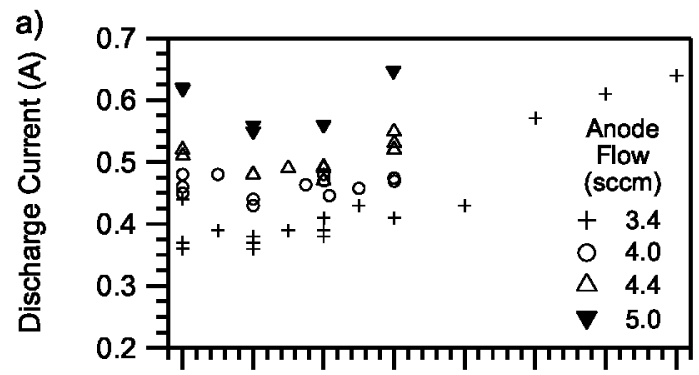

b)
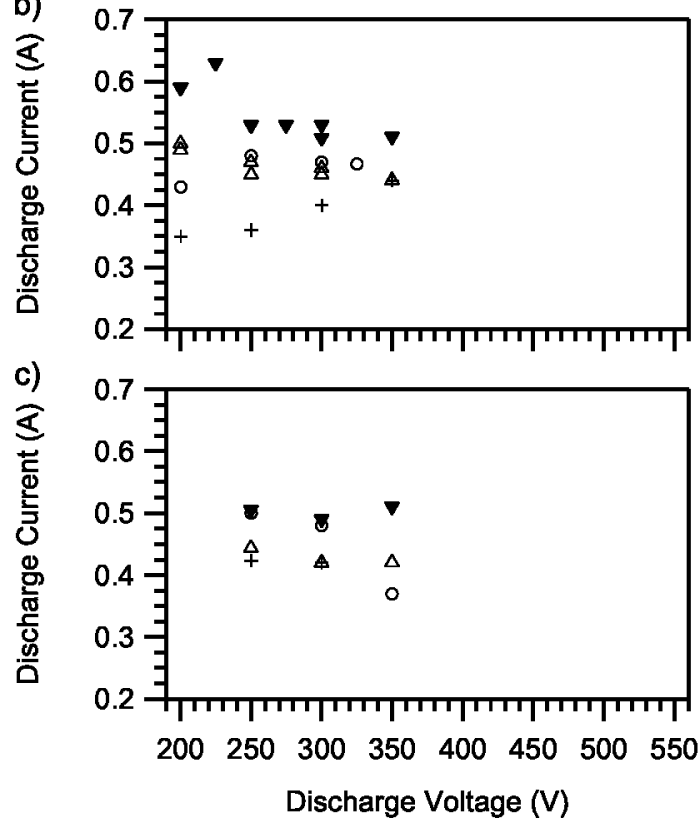

Figure 6. Discharge current as a function of discharge voltage and anode flow rate for the cathode (a) at the base position, (b) linearly translated 1.25-cm (0.5-in) downstream from its base position and (c) linearly translated $2.5-\mathrm{cm}(1.0-i n)$ downstream from its base position.

cusp magnetic field configuration, the current utilization ratio was highest for the direct-field case, indicating it would have a higher efficiency.

Performance measurements on the direct-field magnet configuration thruster over a discharge power level ranging from $70-350 \mathrm{~W}$ and with the cathode orifice located at three different axial positions relative to the thruster exit plane were performed using the MSFC VAHPER thrust stand. The thruster produced thrust levels ranging from 1.25-6.5 mN, anode efficiencies spanning 4-21\%, and $I_{\mathrm{sp}}$ between $400-1950 \mathrm{~s}$. All performance parameters generally increased with discharge power from 140-250 W and did not appear to be a strong function of anode mass flow rate at power levels above $140 \mathrm{~W}$. Above $250 \mathrm{~W}$, the anode efficiency reduced with increasing discharge power. Below $140 \mathrm{~W}$, the data exhibited considerable scatter. At a constant discharge voltage (above $\sim 250 \mathrm{~V}$ ), performance generally increased as the anode mass flow rate was raised from 3.4 to $4.0 \mathrm{sccm}$, but subsequent increases at higher mass flow rates were not readily discernable. A point comparison of the performance of the permanent-magnet thruster and the electromagnet thruster reveal that when the power consumed by the electromagnets is taken into account, the efficiencies of the two thrusters are quite comparable (16-18\% compared to $15.5 \%$, respectively).

\section{Acknowledgments}

Work performed by the Princeton Plasma Physics Laboratory coauthors was partially supported by the U.S. Air Force Office of Scientific Research. We gratefully acknowledge the contributions of MSFC technical support staff Tommy Reid and Doug Galloway, and extend our thanks to students Joseph Balla and Jarred Reneau for their efforts. We also appreciate and acknowledge the continued MSFC support of Mr. James Martin, Mr. J. Boise Pearson, and Ms. Sally Ann Little. 

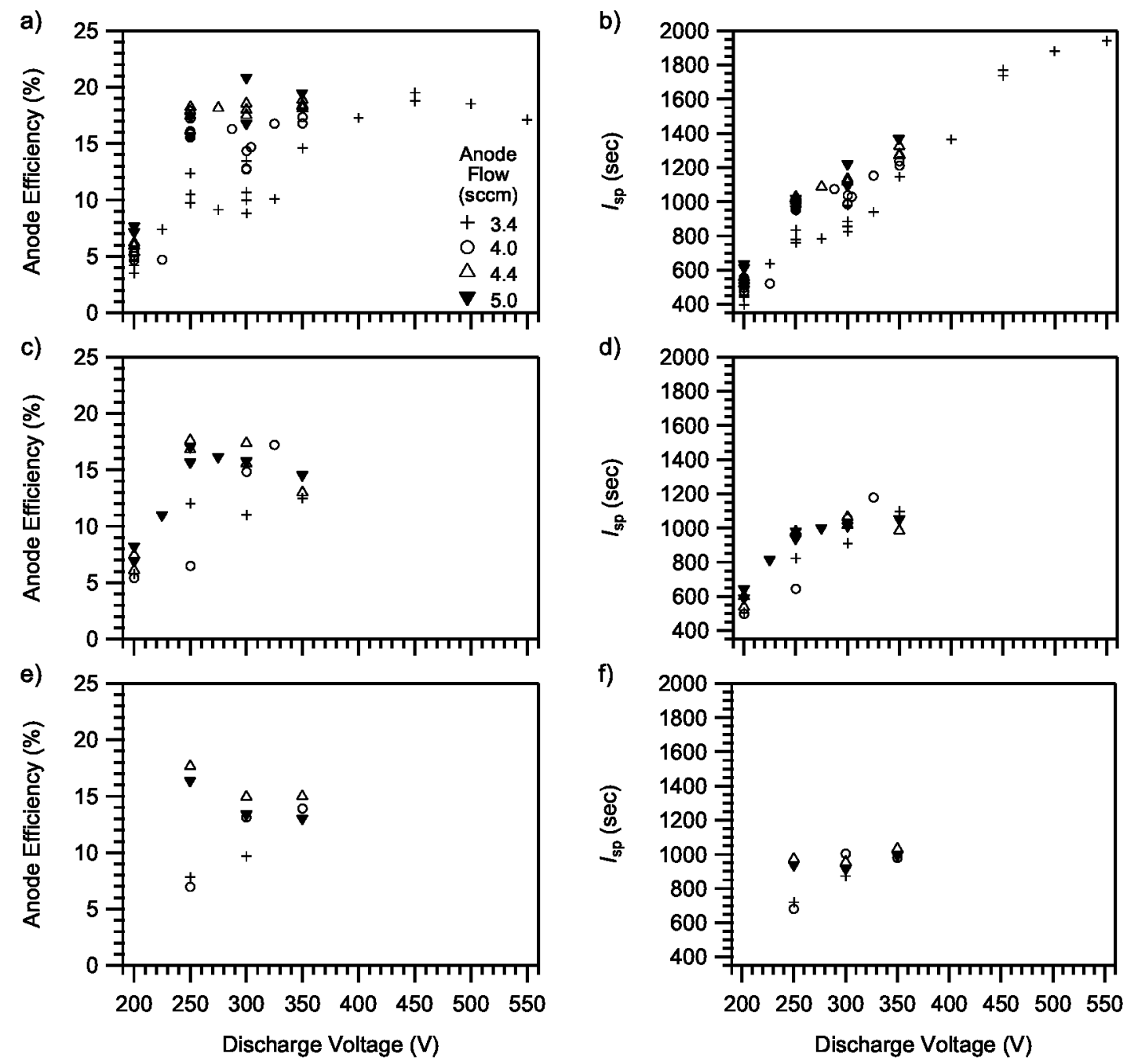

Figure 7. Anode efficiency and $I_{\mathrm{sp}}$ as a function of discharge voltage obtained with the cathode (a,b) at its base position, (a,b) linearly translated $1.25-\mathrm{cm}(0.5-\mathrm{in})$ downstream from its base position, and $(e, f)$ linearly translated $2.5-\mathrm{cm}(1.0-\mathrm{in})$ downstream from its base position.

\section{References}

${ }^{1}$ A.I. Morozov and V.V. Savelyev, "Fundamentals of Stationary Plasma Thruster Theory," Reviews of Plasma Physics, edited by F.F. Kadomtsev and V.D. Shafranov, Vol. 21, Consultants Bureau, New York, 2000, p. 203.

${ }^{2}$ V. Khayms and M. Martinez-Sanchez, "Fifty-Watt Hall Thruster for Microsatellites," Micropropulsion for Small Spacecraft, edited by M.M. Micci and A.D. Ketsdever, Vol. 187, Progress in Astronautics and Aeronautics, AIAA, Reston, VA, 2000, pp. 233-254.

${ }^{3}$ Y. Raitses and N.J. Fisch, "Parametric Investigations of a Nonconventional Hall Thruster," Phys. Plasmas, 8(5):2579, 2001.

${ }^{4}$ A. Smirnov, Y. Raitses, and N.J. Fisch, "Parametric Investigations of Miniaturized Cylindrical and Annular Hall Thrusters," J. Appl. Phys., 92(10):5673, 2002.

${ }^{5}$ A. Smirnov, Y. Raitses, and N.J. Fisch, "Plasma Measurements in a 100 W Cylindrical Hall Thruster," J. Appl. Phys., 95(5):2283, 2004.

${ }^{6}$ K.A. Polzin, T.E. Markusic, B.J. Stanojev, A. Dehoyos, Y. Raitses, A. Smirnov, and N.J. Fisch, "Performance of a Low-Power Cylindrical Hall Thruster," J. Propuls. Power, 23(4):886, 2007.

${ }^{7}$ Y. Raitses, E. Granstedt, A. Smirnov, E. Merino, and N.J. Fisch, "Effects of Cathode Electron Emission on Hall Thruster Discharge," AIAA Paper 2008-5188, July 2008.

${ }^{8}$ Y. Raitses, A. Smirnov and N.J. Fisch, "Enhanced Performance of Cylindrical Hall Thrusters," Appl. Phys. Lett., 90:221502, 2007.

${ }^{9}$ A. Smirnov, Y. Raitses, and N.J. Fisch, "Enhanced Ionization in the Cylindrical Hall Thruster," J. Appl. Phys., 94(2):852, 2003.

${ }^{10}$ Y. Raitses, D. Staack, A. Dunaevsky, L. Dorf, and N.J. Fisch, "Measurements of Plasma Flow in a 2 kW Segmented Electrode Hall Thruster," in 28th International Electric Propulsion Conf., Toulouse, France, 2003. IEPC Paper 03-0139.

${ }^{11}$ K.A. Polzin, T.E. Markusic, B.J. Stanojev, A. DeHoyos and B. Spaun, “Thrust Stand for Electric Propulsion Performance Evaluation," Rev. Sci. Instrum., 77:105108, 2006. 
${ }^{12}$ A. Smirnov, Y. Raitses, and N.J. Fisch, “Electron cross-field transport in a low power cylindrical Hall thruster," Phys. Plasmas, 11(11):4922, 2004.

${ }^{13}$ R.G. Jahn, Physics of Electric Propulsion, McGraw-Hill, New York, 1968, pp. 4, 7. 